\title{
Cloud Computing Intrusion Detection Using Artificial Bee Colony-BP Network Algorithm
}

Yang Hui

SiChuan College of Architectural Technology

Deyang 618000

China

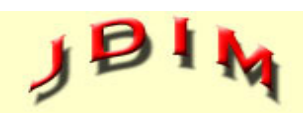

Journal of Digital Information Management
ABSTRACT: Cloud computing characterizes a methodology for computing communications in a much effective manner, and a business paradigm for trading computing resources and services. Alternatively, these difficult and distributed planning's turn a striking objective for intruders. Cloud computing provides huge latent for enhancing production and decrease expenditures. However it simultaneously acquires several novel security risks. Intrusion Detection Systems (IDS) have been employed broadly for identifying malicious actions in network communication and hosts. In this work, an artificial bee colony-BP neural network algorithm is applied to the detection module, in order to detect the complicated aggressive behaviors. Through example verification, the artificial bee colony-BP network algorithm has improved intrusion detection efficiency and classification precision, and can effectively guarantee the safety of the cloud computing environment.

Subject Categories and Descriptors

[D.4.6 Security and Protection] [C.2 Cmputer Communication Networks] Security and protection [F.1.1 Models of Computation]; Neural networks

General Terms: Cloud Computing, Neural Networks, IDS

Keywords: Cloud Computing, Intrusion Detection, BP Neural Network, Artificial Bee Colony Algorithm

Received: 11 January 2018, Revised 1 March 2018, Accepted 12 March 2018

DOI: $10.6025 / \mathrm{jdim} / 2018 / 16 / 4 / 203-209$

\section{Introduction}

With rapid development of internet application and distributed computing technology, cloud computing has become a mature network technology. The cloud computation is designed for integrating multiple calculations and storage resources together for providing virtual sharing computing resource pool for various network services. With continuous development of the cloud computation in modern network technology, the service information safety protections face enormous challenge. An urgent requirement is to identify the ways to improve safety performance of the cloud service. In the meantime, considering that the cloud computing work is different from the heterogeneous virtual environment, traditional single intrusion detection technology is indirectly applied in the cloud computation. This paper introduces a new algorithm, namely artificial bee colony algorithm-BP network algorithm.

\section{Development of Cloud Computing Distributed Intrusion Detection Model}

\subsection{Intrusion Detection Based on Host and Network} The host-based intrusion detection is mainly designed for analyzing and comparing the data record and specified attack model, using the monitoring and analysis host system and application software program log to the obtain the intrusion information. The network-based intrusion detection is mainly designed for monitoring the network data package in the monitoring network segment in realtime; analyze normal and anomaly performance of the data package for processing, with the frame diagram, 


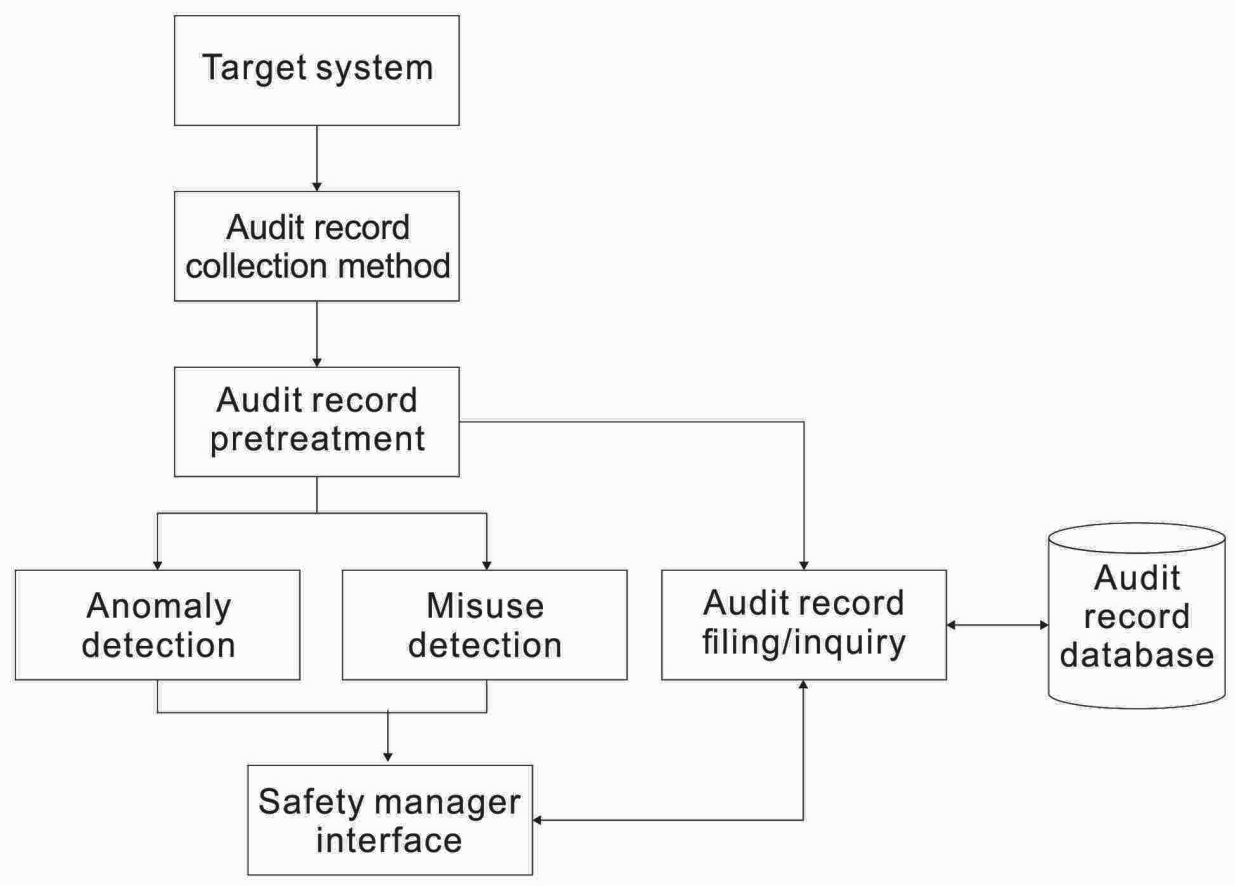

Figure 1. Host-based intrusion detection

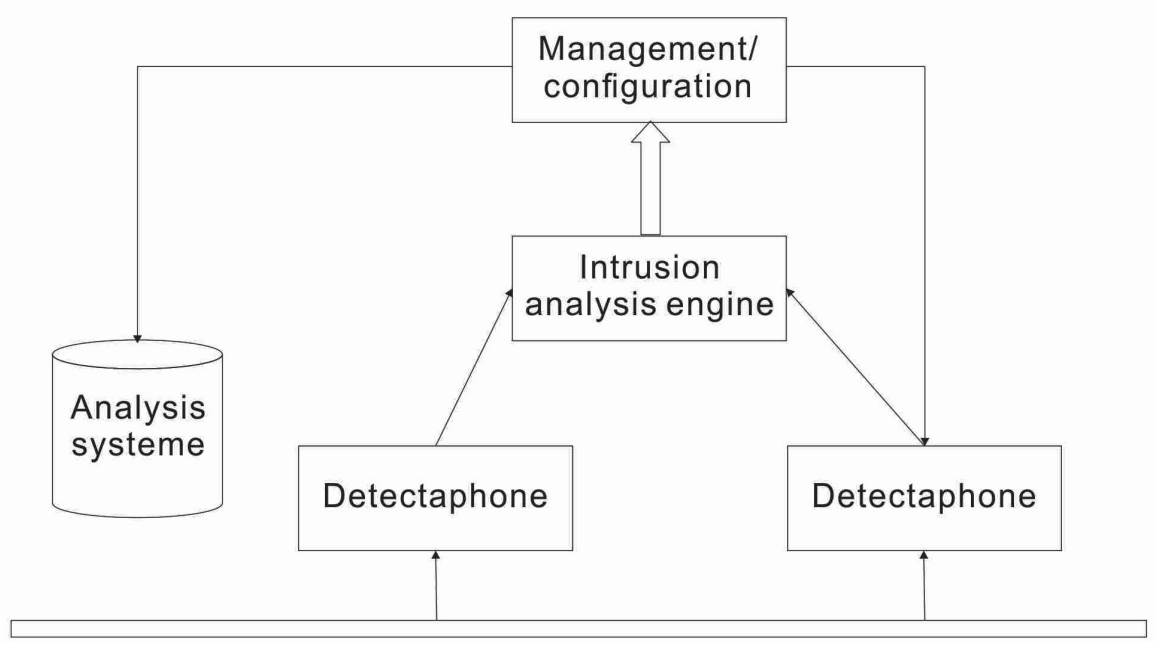

Figure 2. Network-based intrusion detection

\begin{tabular}{|l|l|l|}
\hline & Advantages & \multicolumn{1}{c|}{ Limitations } \\
\hline $\begin{array}{l}\text { Host-based } \\
\text { intrusion } \\
\text { detection }\end{array}$ & $\begin{array}{l}\text { To timely detect and respond to anomaly; } \\
\text { applicable to encoder exchange environment; } \\
\text { without the need of additional software \& hardware }\end{array}$ & $\begin{array}{l}\text { Dependent on the operating platform; } \\
\text { Configuration and computer operation, affecting } \\
\text { host performance; difficult to handle numerous } \\
\text { intrusion activities in the internet environment }\end{array}$ \\
\hline $\begin{array}{l}\text { Network-based } \\
\text { intrusion } \\
\text { detection }\end{array}$ & $\begin{array}{l}\text { The detection doesn't occupy the computer resources, } \\
\text { without affecting the host performance; any local fault } \\
\text { in the network frame will not affect running of ordinary } \\
\text { business; little risk; 24-hour monitoring and } \\
\text { responding to anomaly activities; capable of } \\
\text { detecting failed attack behaviors. }\end{array}$ & $\begin{array}{l}\text { Only able to detect the network segment } \\
\text { information for computer understanding; } \\
\text { network interface adapter with multiple network } \\
\text { intrusion detection system, resulting in high cost. }\end{array}$ \\
\hline
\end{tabular}

Table 1. Comparison of advantages $\&$ disadvantages of both host-based and network-based intrusion detections 


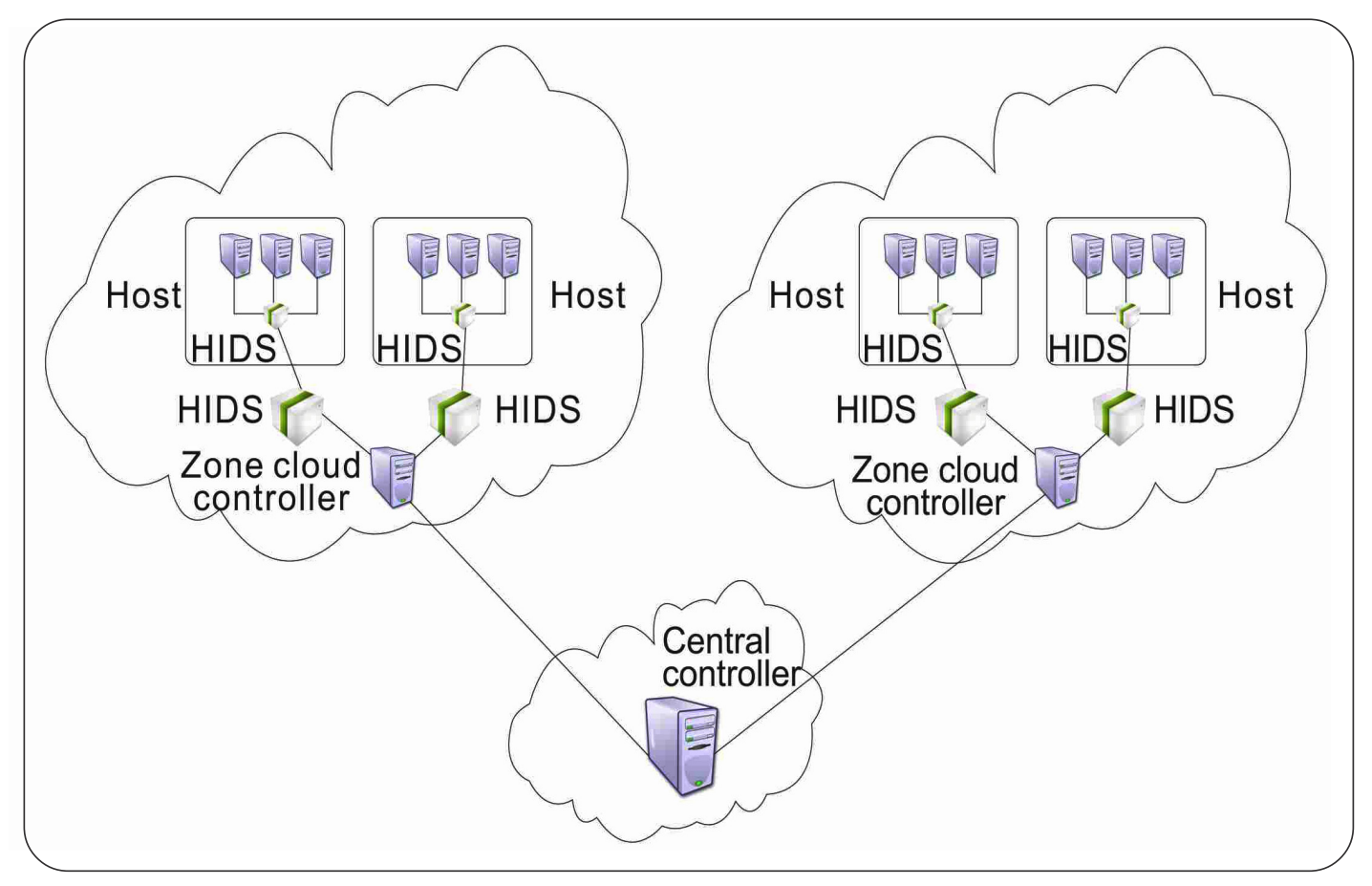

Figure 3. Distributed intrusion detection model

In view of cloud computing isomerization distributed characteristics and complicated unknown attack behavior, the distributed intrusion detection model combined network with host is developed ${ }^{[5-6]}$ which is shown in Figure 3 . In the model, the cloud environment is divided into several modules configured with independent detection agents, and a central controller is used for realizing detection management of each module. The cloud detection system of each module includes the cloud controller, network, and host detection agent, where, the cloud controller is used for analyzing the intrusion information from the network and host detection agent. The detection result is transmitted to the central controller and the central controller is used to realize management dispatching of each module and communication collaboration between modules.

\section{Application Design of Artificial Bee Colony-BP Network Algorithm}

It is necessary to solve complicated and variable attack activities in the cloud environment, which will take long training time for BP neural network, possibly leading to local minimum value, instead of global optimal solution, resulting in great reduction of the system detection efficiency, impossible to guarantee safety of computing environment ${ }^{[7-9]}$. The artificial bee colony algorithm is used for optimizing the weight and threshold of BP neural network, with the resulting values taken as the initial values of the BP neural network, followed by network training operation. The operation is performed alternatively until consistent with the system precision requirements. The process of artificial bee colony-BP network algorithm is as follows:
Step 1: Initialize training sample $P$ of the $B P$ neural network, set the network weight and threshold value, calculate the error function $E$ of actual output value $o_{k}$ of each node and corresponding expected value $T_{k}$, and set the precision.

$$
E=\frac{1}{2} \sum_{p=1}^{p} \sum_{k=1}^{L}\left(T_{k}^{p}-o_{k}^{p}\right)^{2}
$$

Step 2: Initialize the parameter values including minimum value of iteration times Max_Gen and parameter value related to scouter, and set the target function of fitness functions, as well as network weight and threshold value, as the initial solution of $E$ and artificial bee colony algorithm.

Step 3: Equation (2) is used to search the leader and generate new solution $x_{i}^{\text {new }}$ and solve the fitness value. If the new solution is superior to the solution $x_{i}$ for the fitness value, $x_{i}^{\text {new }}$ replaces $x_{i}$, and the retention times is added with 1.

$$
\begin{array}{r}
v_{i j}=x_{i j}+r_{i j}\left(x_{i j}-x_{k j}\right),(k=1,2, \ldots, N, j=1,2, \ldots, d) \\
r_{i j}=\lambda \times \exp \left[\frac{\text { Max_Gen }-G e n}{\text { Max_Gen }}\right]
\end{array}
$$

Where, $N$ and $d$ respectively refer to the number of solutions of the initial population and a random d-dimension vector $x_{i}=(i=1,2, \ldots, N), x_{i j}$ refers to the $j$ th component of the $i$ th solution. $\lambda$, Gen and Max_Gen respectively refer to the fixed constant, current iterations, and maximum iterations.

Step 4: The followers will select the food resource based on selection probability of the roulette and Equation (4), 
followed by corresponding search and calculation in view of the fitness value according to Equation (3);

$$
x_{i j}=x_{i j}^{m i n}+\operatorname{rand}(0,1) \times\left(x_{i j}^{m a x}-x_{k j}^{m i n}\right)
$$

Where, $x_{i j}{ }^{m i n}$ and $x_{i j}{ }^{\text {max }}$ respectively refer to minimum value and maximum value of the $j$ th component of the $i$ th solution.

Step 5: The main purpose of scout operation is to detect whether there is discarded solution, that is to say, the retention times of certain solution increases to "limit", a random solution $x_{i}^{\text {new }}$ is generated according to Equation (4).
Step 6: Run the iteration and store the optimal solution, in order to determine whether Gen is equal to Max_Gen and whether error function $E$ is consistent with specified precision. If either of solutions is met, the operation can stop and the next step can start; otherwise, if Gen = Gen +1 , Step 3 can start. The threshold and weight values are obtained from artificial bee colony algorithm used to substitute for threshold value and initial weight value of the neural network model training of the next loop.

Step 7: Stop the training and obtain the weight and threshold values meeting the conditions, with the flow chart shown in Figure 4.

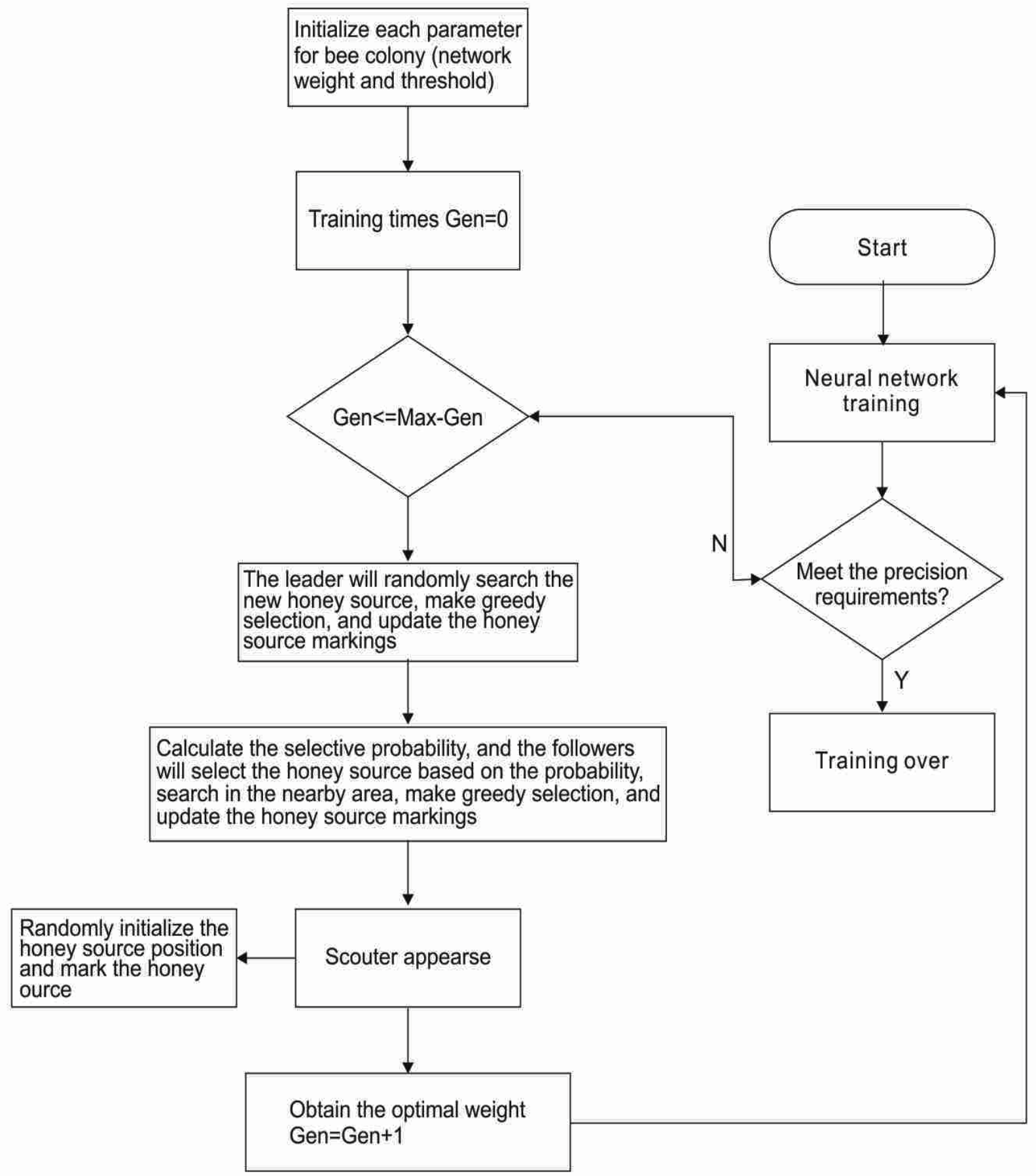

Figure 4. Flow chart of artificial bee colony-BP network algorithm 


\section{Example Simulation Process and Result Analysis}

\subsection{Experiment Process}

KDD CUP99 detecting data is used ${ }^{[10]}$, with the data set including four attack patterns such as denial-of-service attack, unauthorized remote access, scanning and probing, and illegal access to local user ${ }^{[11-15]}$, as shown in Table 2. Other data sets are normal data. The attack pattern of the data is unevenly distributed, with most of attack patterns being DoS attack. In case Probing and DoS attack appear, the normal flow number is basically equal to attack flow number. Generally speaking, the appearance probability of $\mathrm{U} 2 \mathrm{R}$ and $\mathrm{R} 2 \mathrm{~L}$ is small, with little number of attack flows and normal flows, consistent with the conditions for execution of the cloud calculation.

To improve the identification precision of the data set, the pre-treatment operation of the data set shall be completed.

Select the data, as shown in Table 3. Considering that some data characteristics of the data set have micro effect on the experiment result, the data set is subject to dimension reduction operation, for improving the network training speed.

\begin{tabular}{|c|c|}
\hline $\begin{array}{c}\text { Attack } \\
\text { Pattern }\end{array}$ & Specific Attack Pattern \\
\hline Dos & Syn_flood、back、land、Neptune 、 smurf、teardrop、udpstorm \\
\hline U2R & Buffe_overflow、ipsweep、loadmodule、tuscan、rootkit \\
\hline R2L & ftp_wite、password、nmap、multihop、spy、warezmaster , guessing \\
\hline Probing & Port_scanning、nmap、portsweep \\
\hline
\end{tabular}

Table 2. Attack pattern

\begin{tabular}{|c|c|c|c|}
\hline SN & Feature & Description & Type \\
\hline 1 & Duration & Connection duration & Continuity Type \\
\hline 2 & Protocoljype & Protocol for connection & Discrete type \\
\hline 3 & Service & Service type & Discrete type \\
\hline 4 & Flag & Connection status & Discrete type \\
\hline 5 & src_bytes & Number of bits from initiator to receiver & Continuity Type \\
\hline 6 & num_failde_logins & Times of login failure & Continuity Type \\
\hline 7 & num_file_creations & Number of operations of file creation & Continuity Type \\
\hline 8 & Count & $\begin{array}{l}\text { Number of connections with the same target } \\
\text { hosts as the current connection in the last } \\
2 \text { seconds }\end{array}$ & Continuity Type \\
\hline 9 & serror_count & $\begin{array}{l}\text { Number of connections with the same } \\
\text { service as the current connection in the } \\
\text { last } 2 \text { seconds }\end{array}$ & Continuity Type \\
\hline 10 & serror_rate & $\begin{array}{l}\text { Proportion of SYN error connection with the } \\
\text { same target hosts as the current connection } \\
\text { in the last } 2 \text { seconds }\end{array}$ & Continuity Type \\
\hline 11 & srv_serror_rate & $\begin{array}{l}\text { Proportion of SYN error connection with the } \\
\text { same service as the current connection in the } \\
\text { last } 2 \text { seconds }\end{array}$ & Continuity Type \\
\hline 12 & srv_diff_host_rate & $\begin{array}{l}\text { Proportion of connections with the same } \\
\text { service as the current connection but different } \\
\text { target hosts in the last } 2 \text { seconds }\end{array}$ & Continuity Type \\
\hline 13 & dst_host_same_srv_rate & $\begin{array}{l}\text { In the first } 100 \text { connections, proportion of } \\
\text { connections with the same target host and } \\
\text { service as the current connection }\end{array}$ & Continuity Type \\
\hline 14 & Normal or attack & Is it required to attack & Continuity Type \\
\hline
\end{tabular}

Table 3. Selected data 


\begin{tabular}{|l|l|l|l|l|}
\hline Protocol mode & Tep & Udp & Icmp & Unkown \\
\hline Coding & 0 & 1 & 2 & 3 \\
\hline
\end{tabular}

Table 4. Coding of protocol mode

\begin{tabular}{|l|l|l|l|l|l|}
\hline Service mode & Coding & Service mode & Coding & Service mode & Coding \\
\hline Domain_u & 1 & Imap4 & 8 & systat & 19 \\
\hline Ecr_i & 2 & hostnames & 9 & telnet & 17 \\
\hline Eco_i & 3 & netstat & 10 & smtp & 15 \\
\hline ftp & 4 & login & 11 & time & 18 \\
\hline finger & 5 & mtp & 12 & uncp & 19 \\
\hline ftp_data & 6 & other & 13 & Other services & 20 \\
\hline
\end{tabular}

Table 5. Coding of network service mode

\begin{tabular}{|l|l|l|l|l|l|l|l|l|}
\hline Flag & REJ & RSTR & RSTO & SO & S3 & SH & SF & OTHER \\
\hline Coding & 0 & 1 & 2 & 3 & 4 & 5 & 6 & 7 \\
\hline
\end{tabular}

Table 6. Coding of continuous states

\begin{tabular}{|l|l|l|l|l|l|}
\hline & Normal data & DoS & R2L & U2R & Probing \\
\hline Training set & 4000 & 1500 & 500 & 500 & 1500 \\
\hline Testing set & 2000 & 800 & 200 & 200 & 800 \\
\hline
\end{tabular}

Table 7. Data set selection

Process the discrete data continuously, with the protocol, network service, and connection state coder respectively as shown in Table 4, Table 5, and Table 6.

\section{Data Normalization Processing}

Operate the absolute mean square error $S$ :

$$
S=\frac{1}{N} \sum_{i=1}^{N}\left(X_{i}-\bar{X}\right)
$$

Where, $X_{i}$ and $\bar{X}$ respectively represent the data sample and mean value.

Operate the standardized data:

$$
Y_{i}=\frac{X_{i}-\bar{X}}{S}
$$

Considering that the data sample is uniformly normalized to the closed interval $[0,1]$, the linear standard equation used is:

$$
Y=\frac{X-\text { MinValue }}{\text { MaxValue }- \text { MinValue }}
$$

Where, $X$ and $Y$ refers to the value before and after normalization processing; MinValue and Maxvalue respectively refer to data sample minimum and maximum values.

To shorten the neural network training time, 400 data for each set of normal and abnormal data among eight thousand KDD CUP99 detection data set are selected, to complete the training as depicted in Table 7.

In terms of few characteristics of selected data, three nerve cells are selected for the hidden layer, with the number of nerve cells, error, and maximum iterations respectively set as $3,0.01$, and 2000 .

\subsection{Experimental Result}

Both the artificial bee colony-BP network algorithm and 


\begin{tabular}{|l|c|c|c|c|}
\hline & Artificial bee colony-BP network algorithm & \multicolumn{2}{c|}{ BP neural network algorithm } \\
\hline & Detection rate & False alarm rate & Detection rate & False alarm rate \\
\hline Dos & $94.25 \%$ & $3.27 \%$ & $82.35 \%$ & $5.34 \%$ \\
\hline U2R & $88.36 \%$ & $3.55 \%$ & $80.76 \%$ & $6.72 \%$ \\
\hline R2L & $89.25 \%$ & $3.46 \%$ & $81.37 \%$ & $7.31 \%$ \\
\hline Probing & $94.58 \%$ & $2.17 \%$ & $85.62 \%$ & $5.64 \%$ \\
\hline Remarks & $\begin{array}{l}\text { Detection rate = The detected intrusion data/abnormal data samples False alarm rate = Falsely } \\
\text { reported intrusion data/overall data samples }\end{array}$ \\
\hline
\end{tabular}

Table 8. Detection result

BP neural network algorithm are used for detection and the detection results are compared. The results are shown in Table 8.

From Table 7, it is inferred that, the detection rates for DoS and Probing attacks are relatively high, respectively equal to $94.25 \%$ and $94.58 \%$, while those for U2R and $\mathrm{R} 2 \mathrm{~L}$ are low. Due to small amount of attack data for R2L and $U 2 R$, false judgement is prone to occur.

\section{Conclusion}

Through experimental proof, artificial bee colony-BP network algorithm can better identify normal and abnormal data with more advantages than BP neural network algorithm. Artificial bee colony-BP network algorithm can also obtain high detection rate and low false alarm rate. The algorithm can improve the intrusion detection rate and classification precision, and better guarantee safety of the cloud calculation environment.

\section{References}

[1] Cheng Hongxia, Cheng Hongjun. (2011). Study on cloud computation, China New Technologies and Progucts (18) 31-32.

[2] Feng Dengguo, Zhang Min, Zhang Yan, Xu Zhen. (2011). Study on safety of cloud computation, Journal Software, 22 (1) 71-83.

[3] Zhou Xi, Yu Jiong. (2011). Trust-Based defensive system model under cloud calculation, Computer Application, 31 (6)1531-1535.

[4] James, P., Anderson. (1980). Computer security threat monitoring and surveillance, Technical report. James $P$. Anderson Company.

[5] Yanheng, Liu., Daxin, Tian., Xuegang, Yu., et al. (2008).
Large-scale network intrusion detection algorithm based on distributed study, Journal Software, 19 (4) 983-993.

[6] Mu Chengpo. (2011). Network intrusion analysis and intrusion response, Beijing: Beijing Institute of Technology Press, 14-15.

[7] Renshang, Zhang. (2012). Network intrusion detection system based on expert system and neural network, Computer Simulation, 09.162-165.

[8] Dongju, Xu. (2005). Study and realization of intrusion detection system based on neural network. Xinjiang University, 2005

[9] Yangguang, Ou. (2006). Study and realization of network intrusion detection system based on neural network BP algorithm. Southeast University.

[10] DARPA Intrusion detection evaluation. Lexington, MA(USA): Lincoln Laboratory, Massachusetts Institute of Technology, 1999.

[11] Mazzariello, C., Bifulco, R., Canonico, R. (2010). Integrating a network IDS into an open source cloud computing environment, In: 2010 Sixth IEEE International Conference, 265-270.

[12] Xiaodong, Xu., Yanhua, Fan. (2011). DDOS attack intrusion based on macroscopic network flow dependency, Computer Engineering, 37(10) 133-135.

[13] Chuan, Xu., Cheng, Du., Hong, Tang. (2011). Overview of DDoS attack detection study, Study and Development, 85-89.

[14] Ying, Lei., et.al. (2010). DoS/DDoS attack theory and prevention, Microcomputer Information, 8 (3) 77-79.

[15] Wenlong, Li., Kai, Yu., Baosheng, Qu. (2012). Study and realization of Snort-based mixed intrusion detection system, Intelligent Computer and Application, 2095-2101. 